\title{
Subdural haematoma: presentation and diagnosis on medical wards
}

\author{
M. E. WALKER \\ M.A., M.B. Cantab., M.R.C.P. \\ Neurological Registrar
}

Department of Neurology, Leicester Area

\author{
MichaEL ESPIR \\ M.A., M.B. Cantab., M.R.C.P. \\ Consultant Neurologist
}

\author{
R. H. SHEPHARD \\ F.R.C.S. \\ Consultant Neurosurgeon, Regional Neurosurgical Unit, Derby
}

\begin{abstract}
Summary
The twenty-two adult patients with subacute and chronic subdural haematoma seen in Leicester during the last 7 years have been reviewed. The clinical manifestations and results of investigations are described. The diagnostic difficulties which may confront physicians are discussed, with special reference to cases with no history of head injury masquerading as strokes. Stress is laid on the points which help with the differentiation.
\end{abstract}

\section{Introduction}

The diagnosis of subdural haematoma (SDH) does not usually present much difficulty for the neurosurgeon, as once this possibility is considered, it can be readily confirmed by burrhole exploration, which also allows definitive treatment to be started. However, in patients admitted in the first place to general medical rather than neurosurgical wards, and particularly when a history of head injury is lacking, the correct diagnosis may not be suspected initially. In such cases, the differential diagnosis may cover a wide variety of conditions, and the role of the physician is to consider the possibility of SDH and select the suitable cases for investigation and surgical treatment. We have, therefore, reviewed the adult patients with proven SDH in Leicester over the last 7 years, and in this paper report our experiences with special reference to the diagnostic problems encountered.

SDH are now usually classified into acute, subacute and chronic, but the limits of each group vary (McKissock, Richardson \& Bloom, 1960 ; Gilmartin, 1964). We have regarded SDH as acute if operation is performed within 3 days after injury, subacute-more than 3 days but within 3 weeks, and chronic-more than 3 weeks or without any history of head injury (Rosenbluth et al., 1962; Alexander, 1964).
Patients with acute SDH will not be discussed, as they are usually admitted directly to accident or general surgical wards, and deterioration or failure to improve following the recent head injury is a well-recognized indication for burr-hole exploration. Severe brain damage is frequently found coincidentally, and evacuation of the SDH may make little difference to the ultimate outcome.

On the other hand, with subacute and chronic $\mathrm{SDH}$ the correct diagnosis is often not obvious and yet most vital, as these patients often have relatively little associated brain damage and timely surgical treatment is much more rewarding.

In this paper we first describe the clinical manifestations and preliminary investigation of the patients with subacute and chronic SDH to indicate the common features which can be applied to help with the diagnosis of the difficult cases. This latter group will then be discussed separately in order to illustrate the particular diagnostic difficulties and emphasize their distinguishing features.

\section{Clinical manifestations}

There were twenty-two patients in this series, nine with subacute and thirteen with chronic SDH. All recovered after surgical treatment, except two of the chronic cases. Table 1 shows that in thirteen cases (eight subacute and five chronic) there was a clear-cut relation to a head injury before the diagnosis was established by burr-holes, but in the remaining nine cases (eight chronic and one subacute) no such history was obtained, or a previous head injury was either trivial, remote or of doubtful significance, so that the diagnosis of SDH was not obvious and not suspected initially.

\section{Predisposing factors}

The liability of alcoholics to develop SDH is well known (Brain, 1962), and this has also been reported in epileptics (Feldman, Pincus \& 
TABLE 1

History of head injury

\begin{tabular}{lccc}
\hline & Definite & $\begin{array}{c}\text { Doubtful } \\
\text { or trivial }\end{array}$ & Nil \\
\hline Subacute & 8 & 1 & 0 \\
Chronic & 5 & 1 & $7(3)$ \\
\hline
\end{tabular}

The number of cases of subacute and chronic SDH with a history obtained before exploration of definite, doubtful or trivial, and no head injury. The figure in parentheses indicates that in three of the seven cases with no head injury before exploration, a retrospective history of head injury was obtained after exploration.

McEntee, 1963). Of the twenty-two patients in this series, three were alcoholic and sustained their head injuries while drunk, and another three patients were epileptic and sustained their head injuries during fits.

Other known causes, such as ruptured intracranial aneurysm and angioma, intracranial tumours, blood dyscrasias and infections (Logue, 1951) and anticoagulant therapy (Wiener \& Nathanson, 1962 ; Strang \& Tovi, 1962) were not encountered in this series.

\section{Symptoms and signs}

The cardinal clinical features were: (1) headache, (2) drowsiness, (3) lateralizing neurological signs, and (4) pupillary abnormalities. These usually occurred in various combinations, although in two patients headache was the sole presenting feature.

(1) Headache. Headache was a predominant presenting symptom in all sixteen patients in whom an adequate history could be obtained. In the twelve patients who gave a history of headache and head injury, the headache dated from the injury in eight, and in the remaining four there were symptom-free intervals ranging from 2 days to 4 months. In three patients the headache was particularly severe at night or on waking suggestive of raised intracranial pressure, and one of these had papilloedema. Five other patients also had papilloedema; three of these were drowsy or confused on admission and no history was obtainable, but the other two were interesting as they both had unilateral headache and in each case this was on the side of the SDH. One patient consistently woke free of headache, but would develop it immediately he got out of bed. This symptom suggested a low intracranial pressure, which was confirmed at lumbar puncture.

(2) Drowsiness. Twenty of the twenty-two patients were drowsy on admission, and six showed definite fluctuations in the level of consciousness. Four patients were deeply unconscious by the time they underwent surgery. In the two patients who were not drowsy, headache was the main complaint.

\section{(3) Lateralizing neurological signs}

Hemiparesis. Seventeen patients had a hemiparesis, which was usually mild, particularly when compared to the degree of depression of consciousness. Thus the hemiparesis was slight in fifteen and moderate in two, and no patient had a complete hemiplegia. Yet all these patients had depression of consciousness ranging from drowsiness to coma. In seven of these seventeen patients the hemiparesis was ipsilateral to the SDH.

Bilateral extensor plantar responses were found in six patients, but all were unconscious at the time of this finding.

Dysphasia. In the eight patients who were definitely dysphasic, the SDH was on the left side and all were right-handed.

Hemianaesthesia and hemianopia. These signs were found in only one patient, and cleared after evacuation of the SDH. Although drowsiness may mask these two signs, nevertheless they are uncommon in SDH.

(4) Pupillary abnormalities. Inequality of the pupils with variations in size was noted in four patients. The SDH was found on the side of the larger pupil in three patients, and on the opposite side in one.

\section{Preliminary investigations}

Three investigations discussed here are: (1) plain X-rays of the skull, (2) electroencephalography (EEG), and (3) lumbar puncture. (Although echoencephalography and isotope scanning may also be useful, these techniques were available for only a few cases in this series.)

\section{(1) Skull X-rays}

Twenty patients had plain X-rays taken of the skull; in ten the pineal gland was seen to be calcified, and significant lateral displacement was shown in five. A fracture of the skull was seen in five cases, of which four had subacute SDH, and in the other the SDH was evacuated on the $21 \mathrm{st}$ day after injury. In one patient both pineal displacement and a fracture were present.

Thus the skull X-rays showed abnormalities in nine out of the twenty patients. Table 2 relates the side of the SDH and direction of lateral pineal displacement to the side of the hemiparesis when present. It will be seen that of the four patients with a hemiparesis, this was contralateral in three, but in case A.P., the direction of pineal displacement indicated that the intracranial lesion was ipsilateral to the hemiparesis, a feature which suggested the diagnosis of SDH. 
TABLE 2

The side of the SDH and direction of the lateral pineal displacement in relation to the side of the hemiparesis (when present) in the five patients in whom shift of the calcified pineal was shown on the plain skull X-rays

\begin{tabular}{lccc}
\hline Case & $\begin{array}{c}\text { Side of } \\
\text { SDH }\end{array}$ & $\begin{array}{c}\text { Direction of } \\
\text { pineal shift }\end{array}$ & $\begin{array}{c}\text { Side of } \\
\text { hemiparesis }\end{array}$ \\
\hline J.C. & $\mathbf{R}$ & to $\mathrm{L}$ & $\mathrm{Nil}$ \\
A.W. & $\mathrm{R}$ & to $\mathrm{L}$ & $\mathbf{L}$ \\
B.H. & $\mathrm{L}$ & to $\mathrm{R}$ & $\mathrm{R}$ \\
C.B. & $\mathrm{L}$ & to $\mathrm{R}$ & $\mathrm{R}$ \\
A.P. & $\mathrm{L}$ & to $\mathrm{R}$ & $\mathbf{L}$ \\
\hline
\end{tabular}

\section{Electroencephalography}

The EEG was recorded in seven of our patients (six chronic and one subacute SDH). The EEG abnormalities indicated the side of the SDH in six, but in the other there was bilateral fast activity of low voltage in a patient who had a large left sided SDH. Table 3 shows the side of the SDH and EEG abnormality related to the side of the

TABLE 3

The side of the SDH and EEG abnormality in relation to the side of the hemiparesis (when present) in the seven patients who had the EEG recorded

\begin{tabular}{lccc}
\hline Case & $\begin{array}{c}\text { Side of } \\
\text { SDH }\end{array}$ & $\begin{array}{c}\text { Side of EEG } \\
\text { abnormality }\end{array}$ & $\begin{array}{c}\text { Side of } \\
\text { hemiparesis }\end{array}$ \\
\hline J.C. & R & R & Nil \\
W.B. & L & Bilateral & Nil \\
P.C. & L & L & Nil \\
A.B. & L & L & Nil \\
B.H. & L & L & R \\
W.N. & L & L & R \\
R.K. & L & L & L \\
\hline
\end{tabular}

hemiparesis when present. It will be seen that of the three patients with a hemiparesis, this was contralateral in two, but in case R.K. it was ipsilateral, so suggesting the diagnosis of SDH.

\section{(3) Lumbar puncture}

Sixteen of the patients had a lumbar puncture, and Table 4 shows the time interval between this and the head injury (when known) and the results. It can be seen that in eleven of the sixteen cases the cerebro-spinal fluid (CSF) was normal in content, and that-with one exception-it was only blood stained or xanthochromic in patients known to have had a recent head injury (i.e. within 2 weeks). Furthermore, the white count and protein content were only raised in fluids that were xanthochromic or blood stained, and such evidence of traumatic subarachnoid haemorrhage from the CSF constituents was of little help with the diagnosis of SDH. However, the pressure of CSF was of considerable value, being high (over $200 \mathrm{~mm}$ ) in three cases and so clearly indicating the need for further investigations, which duly revealed the diagnosis. In two cases, the CSF pressure was low (50 and $30 \mathrm{~mm}$ ), as not infrequently occurs in chronic SDH and in these two cases it was this finding which first suggested the true diagnosis.

\section{The difficult cases}

Nine patients presented particular diagnostic difficulties. In two this was because the head injury was trivial or of doubtful significance, and in seven because there was initially no history of head injury. In three of this latter group, a history of head injury was eventually obtained, but only after the SDH had been evacuated.

TABLE 4

The time interval between the lumbar puncture and the head injury (when known), together with the CSF findings in the sixteen patients on whom lumbar puncture was performed

\begin{tabular}{|c|c|c|c|c|c|c|}
\hline Case & Interval & $\begin{array}{l}\text { Pressure } \\
(\mathrm{mm})\end{array}$ & $\begin{array}{c}\text { Protein } \\
(\mathrm{mg} / 100 \mathrm{ml})\end{array}$ & $\begin{array}{r}\mathrm{WBC} \\
\left(\mathrm{mm}^{3}\right)\end{array}$ & $\begin{array}{r}\mathrm{RBC} \\
\left(\mathrm{mm}^{3}\right)\end{array}$ & Colour \\
\hline F.Bc & 2 days & 150 & 100 & 40 & 62,000 & Blood-stained \\
\hline J.C. & 5 days & 'normal' & 80 & $?$ & 2,500 & Blood-stained \\
\hline A.B. & 6 days & 70 & 140 & 10 & 5,150 & Xanthochromic \\
\hline C.B. & 13 days & 140 & 40 & 0 & 30 & Xanthochromic \\
\hline A.P. & 14 days & 80 & 30 & $\mathbf{0}$ & 0 & Colourless \\
\hline R.K. & 16 days & 170 & 10 & 0 & 20 & Colourless \\
\hline V.B. & 18 days & 185 & 50 & $?$ & 388 & Colourless \\
\hline R.N. & 6 weeks & 240 & 30 & 0 & 0 & Colourless \\
\hline B.H. & 2 months & 75 & 30 & 0 & 0 & Colourless \\
\hline N.B. & 6 months & 130 & 20 & 0 & 2 & Colourless \\
\hline R.O. & 6 months & 190 & 40 & 5 & 0 & Colourless \\
\hline P.C. & & 300 & 30 & 2 & 3 & $\begin{array}{l}\text { Very slightly } \\
\text { xanthochromic }\end{array}$ \\
\hline W.J. & & 270 & 30 & 3 & 0 & Colourless \\
\hline F.B. & & 170 & 20 & 1 & 0 & Colourless \\
\hline C.F. & & 50 & 40 & 0 & 0 & Colourless \\
\hline A.W. & & 30 & 'Normal' & 0 & 0 & Colourless \\
\hline
\end{tabular}


The following case histories illustrate some of the problems encountered. In the first four cases a stroke was the initial diagnosis.

\section{Case 1}

V.B., a 57 year-old-woman, fell at the bottom of some stairs and injured her head, losing consciousness for just a few minutes. During the next 2 weeks she had intermittent stiffness of her right upper limb and slight difficulty with her speech. She was then admitted to hospital, and later that day became disorientated with a right hemiparesis and bilateral extensor plantar responses. She became increasingly drowsy and the right pupil became fixed and dilated. Lumbar puncture showed colourless CSF under pressure of $185 \mathrm{~mm}$, there were 388 red cells and the protein was $50 \mathrm{mg}$ / $100 \mathrm{ml}$. Plain X-rays of the skull showed no abnormality, but a left carotid arteriogram revealed a left sided subdural collection, which was successfully evacuated.

Comment. In this case the significance of the head injury was initially uncertain, and it seemed possible that the fall and subsequent signs resulted from cerebro-vascular insufficiency. However, the bilateral extensor plantar responses, deepening coma and later the dilated and fixed right pupil suggested tentorial herniation rather than unilateral cerebral ischaemia, and the true significance of the head injury then became clear. However, post-operatively her dysphasia did not clear completely, and a right homonymous hemianopia was noted; it seems possible that she may have in fact sustained a cerebro-vascular lesion at the onset, leading to her fall and the development of the SDH.

\section{Case 2}

N.B., a 56-year-old man, had a previous history of both cerebral and myocardial infarctions. Seven days before admission he developed severe headache associated with weakness of the right lower limb. These symptoms increased and on the day of admission he also developed weakness of the right arm and dysphasia. Lumbar puncture showed clear CSF under normal pressure with normal constituents. Plain X-rays of his skull showed no abnormality. He later became increasingly drowsy without any increase in the hemiparesis, and it was this that raised the possibility of a SDH. Burrholes were performed and a large left-sided SDH was successfully evacuated. Subsequently it was learnt that he had sustained a minor head injury 6 months previously.

Comment. Taking into account his past history, it was thought-not unnaturally-that he had suffered a further cerebral infarct. However, the following points were more in favour of SDH than cerebral infarction: (a) the headache was severe $\vec{\nabla}$ and persistent, (b) the hemiparesis started and was $\stackrel{\square}{\square}$

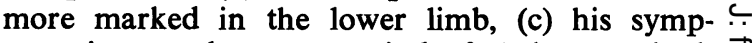
toms increased over a period of 7 days, and (d) finally there was increasing drowsiness without increase in the hemiparesis, which is a characteristic feature of SDH.

\section{Case 3}

B.H. a 71-year-old man, was admitted in a con- के fused and drowsy state with no available history. $\vec{\circ}$ Examination revealed a mild right hemiparesis with dysphasia, and left cerebral infarction was the initial diagnosis. Lumbar puncture showed clear CSF under normal pressure with normal constituents. EEG showed generalized slowing with lower voltage on the left. However, pineal displacement to the right was noted on the plain skull $\mathrm{X}$-rays and $\mathrm{or}$ burr-holes were therefore advised. These revealed $T$ a left-sided SDH which was removed, leading to complete recovery. Only afterwards was it learnt that the patient had suffered a minor head injury at work 2 months previously.

Comment. This patient also exhibited marked drowsiness with only a slight hemiparesis, and in addition the pineal was displaced.

\section{Case 4}

A.W., a 66-year-old man, developed episodes of dizziness and headache with slight left hemiparesios After approximately $48 \mathrm{hr}$ he lapsed into unconsciousness, and as his general practitioner thought that he had a stroke he was nursed at home for the next 5 days. His admission to hospital was then requested for terminal care, and on examination he was deeply unconscious and moribund. Lumbar puncture revealed clear CSF under a low pressure of $30 \mathrm{~mm}$ with normal constituents. The possibility of a SDH was queried because of the low CSF pressure, and arrangements were made for him to have plain X-rays taken of the skull followed by burr-holes. These revealed a right sided chronic SDH which was evacuated, and he eventually recovered completely.

Comment. His skull X-rays in fact showed that the pineal gland was calcified and displaced to the left, but the decision had been made to proceed with exploratory burr-holes before the radiological findings were known, because of the low CSF pressure. Questioned carefully in retrospect, he had no recollection of any head injury.

In the following two cases, an intracranial tumour was the initial diagnosis.

\section{Case 5}

F.B., a 60-year-old man, had 1 month's history 
of progressive right hemiparesis and dysphasia. There was no history of head injury. Lumbar puncture showed clear CSF under normal pressure with normal constituents. Subsequently he developed fluctuating drowsiness and papilloedema. Cerebral angiography showed a left sided subdural collection, and evacuation was followed by complete recovery.

Comment. He was at first thought to have a cerebral tumour because of the history of progressive hemiparesis, and this was the indication for arteriography which demonstrated the correct diagnosis. The possibility of a SDH had also been raised by the fluctuating drowsiness.

\section{Case 6}

R.O., a 51-year-old man, had a 6-month history of severe headache exacerbated by neck movements, and a 2-week history of drowsiness and incontinence of urine. The only other abnormal neurological sign was slight hypotonia of the left arm. Plain X-rays of the skull showed no abnormality. Following lumbar puncture (which revealed normal CSF under a pressure of $190 \mathrm{~mm}$ ) he became deeply unconscious with bilateral extensor plantar responses, but soon reverted to his former state. Several similar fluctuations of his level of consciousness recurred during the next $48 \mathrm{hr}$, and exploratory burr-holes then revealed a left sided chronic SDH. Unfortunately, the brain failed to re-expand post-operatively and he died. A history of two minor head injuries 6 months previously was obtained in retrospect from his wife.

Comment. The marked drowsiness, bilateral extensor plantar responses, and the deterioration following lumbar puncture made one suspect tentorial herniation due to a space-occupying lesion. Its exact nature was uncertain, but the fluctuating course and minimal signs were suggestive of a SDH.

In the following two cases there was evidence of raised intracranial pressure without lateralizing or other diagnostic clinical signs.

\section{Case 7}

W.J., a 52-year-old man, had a history of frontal headache and nasal catarrh for 2 weeks. Sinus Xrays had shown an opaque right antrum, and this was washed out. However, severe headache continued and so he was admitted for further investigations. During the next few days he became drowsy, but there were no other neurological abnormalities. Lumbar puncture showed normal CSF, but the pressure was raised to $270 \mathrm{~mm}$. The day after lumbar puncture he became deeply unconscious with periodic breathing, constricted pupils and extensor plantar responses. Burr-holes revealed a large right sided SDH which was successfully evacuated.

Comment. There was no history of head injury, but the raised CSF pressure and the serious deterioration following lumbar puncture led to the diagnostic and therapeutic exploration.

\section{Case 8}

P.C., a 20-year-old youth, developed sudden left frontal headache immediately after diving into a swimming pool. He did not bang his head and there was no loss of consciousness. The headache persisted for 1 week and was associated with frequent vomiting. There followed an asymptomatic period of 2 months, after which his previous symptoms returned. He was admitted to hospital a week later, and on examination he was alert with no abnormal neurological signs. Lumbar puncture showed normal CSF under a raised pressure of $300 \mathrm{~mm}$. Plain $\mathrm{X}$-rays of the skull showed no abnormality. Later mild papilloedema developed, and it was noted that the resonance of the percussion note differed on the two sides of the skull (Macewen's sign). EEG showed irregular slow waves on the left, and a carotid arteriogram revealed a large left-sided subdural collection which was successfully evacuated.

Comment. In this patient there was evidence of a space-occupying intracranial lesion, the EEG abnormality indicating that this was left-sided and supratentorial. The positive Macewen's sign was also consistent with the possibility of a SDH. The absence of drowsiness and of lateralizing neurological signs were notable features in this case. The possible precipitation by exertior or strain rather than any actual head injury is also of interest, for despite repeated questioning this patient persistently denied striking his head on the bottom of the pool at the onset, or any other head injury.

The following case was of special interest and proved to be one of the most difficult in this series.

\section{Case 9}

C.F., a 56-year-old man, gave a 2-year history of headache and dizziness, and occasional vomiting. His first attack of headache occurred while he was bending down working on his bicycle. There was no history of head injury. He had moderate hypertension, blood pressure being 180/ $120 \mathrm{mmHg}$, but despite treatment with methyldopa, his headache had not improved and there had been no response to simple analgesics. Each morning he would wake free of headache, but standing would immediately bring it on. He even- 
tually developed intermittent drowsiness, with hiccoughs and frequent vomiting, although when he was admitted to hospital there were no other neurological abnormalities. Plain X-rays of the skull were normal, and lumbar puncture showed normal CSF but a low pressure of $50 \mathrm{~mm}$. Carotid angiograms revealed bilateral subdural collections. Unfortunately the brain failed to re-expand after these had been evacuated, and the patient died.

Comment. The low pressure state was the salient feature suggesting the diagnosis of SDH in this case. In retrospect it seemed possible that the strain from bending down when working on his bicycle precipitated the haemorrhage.

\section{Discussion}

The case histories cited above illustrate certain characteristic features of SDH, some of which can be correlated with the pathological processes involved. The initial incident may be the rupture of one or more of the veins traversing the subdural space between the cerebral hemisphere and the superior sagittal sinus. Such an event seems more likely to occur in patients with cerebral atrophy, or in those subject to frequent head injuries (e.g. epileptics and alcoholics). In many cases a head injury is known to be the precipitating cause, but in most series there is a group of patients from whom no history of head injury can be obtained. Although in such cases a trivial head injury may have been forgotten, it seems possible that SDH can occasionally result from just coughing, straining or exertion. It has also been suggested that in the absence of any known history of head injury, spontaneous bleeding and haematoma formation may occur intradurally, i.e. between the inner and outer layer of the dura, possibly due to a congenital diathesis (Jewesbury \& Josse, 1964).

The haematoma overlying the cerebral cortex may enlarge as a result of an osmotic mechanism (Gardner, 1932). It may then cause the percussion note of the skull to be flatter on the affected side (Macewen's sign), although this may be difficult to elicit and is not pathognomonic of SDH. In some cases the haematoma is of maximal thickness in the parasagittal region, so that the hemiparesis may start and be most severe in the lower limb, as described in one of our cases. Although this may also result from infarction in the territory of the anterior cerebral artery, this is relatively rare compared with infarction in the distribution of the middle cerebral artery, when the upper limb is mainly affected. Furthermore with superficial parietal lesions, hemianaesthesia and hemianopia would not be expected, whereas they are of course common in cerebral infarction and other more deeply situated lesions.
SDH occasionally become calcified or ossified (Jackson \& Clare, 1965), sometimes they may re-z gress spontaneously (Bender, 1960; Ambrosetto, $\stackrel{\mathbb{Q}}{\Omega}$ 1962 ; Lancet, 1962), but the majority graduallyc. expand, compressing the underlying brain and its $=$ venous drainage. Eventually this may cause the? medial part of the ipsilateral temporal lobe to herniate through the tentorial hiatus. The third흠 nerve may thus be compressed, and inequality with $\frac{\text { }}{\widetilde{\sigma}}$ fluctuation in size of the pupils is a well-known sign in SDH. At the same time, the opposite cerebral peduncle may be compressed against the $-\overrightarrow{0}$ tentorial edge, causing a hemiparesis on the same side as the SDH. In this series the frequency with $\vec{\omega}$ which the hemiparesis was ipsilateral to the $\mathrm{SDH}_{0}^{\circ}$ has been mentioned. Although this phenomenono may also occur in some intracranial tumours (e.g. meningioma), nevertheless this is of diagnostic value in the differentiation of SDH from cerebralor infarction. Pre-operative evidence regarding the $\vec{\sigma}$ lateralization of the SDH may be obtained by noting lateral pineal displacement on the plain skull ${ }_{\circ}^{\circ}$ $X$-rays, and/or predominantly unilateral EEG abnormalities. Although the EEG is usually $\vec{O}$ abnormal and may be helpful, particularly if the abnormality is ipsilateral to the hemiparesis, never-응

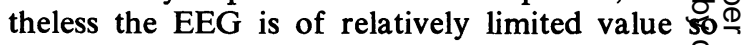
that if not readily available this should not lead $\mathbb{0}_{\overrightarrow{0}}$ any undue delay of other necessary measures.

The clinical, radiological and EEG findings main thus indicate the correct diagnosis and lateraliz $\mathrm{a}^{+}$ tion. However, as far as treatment is concerned, as SDH may be bilateral whether or not lateralizing signs are present, and even if SDH is found and $\frac{O}{\mathbb{D}}$ evacuated from one side, burr-hole exploration $\stackrel{\square}{\rightarrow}$ should always be done on both sides.

As with other intracranial space-occupying lesions, the manifestations of SDH result from both its local effects on underlying cerebral tissues and its general effects on intracranial $\frac{1}{3}$ dynamics. However, because the clot is extracerebral and often exerts its effects over a wide 3 area of cerebral cortex, symptoms and signs of local pressure (e.g. hemiparesis) tend to be relatively slight, whereas those of generalized dis- 0 turbance of intracranial dynamics (e.g. headache, drowsiness or coma) tend to be severe. These $\frac{D}{0}$ features contrast sharply with those of cerebral infarction, when a lesion of sufficient magnitude $N$ to produce drowsiness is usually associated with a $N$ dense hemiplegia, and often hemianaesthesia and $N$ hemianopia as well.

The main difficulties in diagnosis occur when there is no known history of head injury, or when the significance of a minor or remote head injury is uncertain. In these patients the initial diagnosis ? is usually cerebral infarction or tumour. The sus- 
picion of cerebral tumour usually merits further investigations, so that the correct diagnosis is revealed. However, this does not hold true in suspected stroke. This problem frequently arises in the elderly (Bedford, 1957; Stuteville \& Welch, 1958 ; Perlmutter, 1961 ; Spencer, 1964), in whom mental changes tend to be attributed to arteriosclerosis and when falls and minor head injuries are commonplace, so that even if there is a history of such an injury its true significance may not be appreciated. Furthermore, because of old age, arteriosclerosis or hypertension, cerebral angioraphy may be withheld, and thus the true diagnosis may not even be revealed by chance. It is clearly impracticable and undesirable to perform burrholes or arteriograms on all cases of suspected stroke, but the features which we have discussed may indicate the necessity for proceeding with these investigations.

\section{Conclusions}

Although this is a small series of cases, we feel justified in emphasizing that the diagnosis of $\mathrm{SDH}$, especially in the difficult cases with no history of head injury, should be considered:

(a) When there is drowsiness or coma, or evidence of raised intracranial pressure without any other obvious signs or cause, particularly in an epileptic or alcoholic.

(b) In suspected cerebral infarction: (1) when there is severe or persistent headache, (2) when the level of consciousness and physical signs fluctuate in severity, (3) when depression of consciousness is marked with only a slight hemiparesis, (4) when the hemiparesis affects the leg more than the arm, (5) when there is inequality and fluctuation in size of the pupils, (6) when the percussion note of the skull differs on the two sides, (7) when the pressure at lumbar puncture is abnormally low or high, (8) when plain skull X-rays show a fracture or pineal shift, and (9) when plain skull X-rays (echo-encephalography if available), and/or EEG indicate an intracranial lesion on the same side as the hemiparesis.

Proof of the diagnosis of SDH and successful treatment are dependent on timely burr-hole exploration, and although arteriography may also be a most valuable diagnostic aid, yet these procedures demand valid indications. When they are readily fulfilled the prognosis should be excellent, but in difficult cases the indications for these procedures may depend upon paying attention to the details discussed, and recovery may then result from an otherwise hopeless situation.

\section{Acknowledgments}

We wish to acknowledge with thanks the collaboration of our colleagues who have helped with the management of the cases reported.

\section{References}

Alexander, J.B. (1964) Chronic subdural haematoma, as seen by the internist. N.Carolina med. J. 25, 95.

Ambrosetro, C. (1962) Post-traumatic subdural haematoma : further observations on non-surgical treatment. Arch. Neurol. 6, 287.

BEDFORD, P.D. (1957) Intracranial haemorrhage-diagnosis and treatment. Proc. R. Soc. Med. 51, 209.

BENDER, M.B. (1960) Recovery from subdural haematoma without surgery. J. Mt Sinai Hosp. N.Y. 27, 52.

BraIn, LoRd (1962) Diseases of the Nervous System, Sixth edition, p. 309. Oxford University Press, London.

Feldman, R.G., Pincus, J.H. \& McEnTee, W.J. (1963) Cerebrovascular accident or subdural fluid collection? Arch. intern. Med. 112, 966.

GARDNER, W.J. (1932) Traumatic subdural haematoma: with particular reference to the latent interval. Arch. Neurol. 27, 847.

Gilmartin, D. (1964) Arteriography in diagnosis of subdural haematoma. Lancet, i, 1061.

JACKsON, F.E. \& ClARE, F. (1965) Ossified subdural haematomas of the cerebral convexities. J. Amer. med. Ass. 191, 598.

JeWeSbury, E.C.O. \& Josse, S.E. (1964) Arteriography in diagnosis of subdural haematoma. Lancet, i, 1277.

Lancet (1962). Annotation, ii, 1154.

Logue, V. (1951) Chronic subdural effusions. Modern Trends in Neurology (Ed. by A. Feiling), p. 363. Butterworth, London.

McKissock, W., Richardson, A. \& Bloom, W.H. (1960) Subdural haematoma: A review of 389 cases. Lancet, i, 1365.

Perlmutter, I. (1961) Subdural haematoma in older patients. J. Amer. med. Ass. 176, 212.

Rosenbluth, P.R., Arias, B., Quartetti, E.V. \& Carney, A.L. (1962) Current management of subdural haematoma: analysis of 100 consecutive cases. J. Amer. med. Ass. 179, 759.

SPENCER, W. (1964) Problems in diagnosis of intracranial disease among the aged. J. Mt Sinai Hosp. N.Y. 31, 17.

Strang, R.R. \& Tovi, D. (1962). Subdural haematomas complicating anticoagulant therapy. Brit. med. J. i, 845.

Stuteville, P. \& Welch, K. (1958) Subdural haematoma in the elderly person. J. Amer. med. Ass. 168, 1445.

Wiener, L.M. \& Nathanson, M. (1962) The relationship of subdural haematoma to anticoagulant therapy. Arch. Neurol. 6, 282. 